\section{Neurosyphilis with normal pressure hydrocephalus and dementia paralytica: serial clinical, laboratory and radiological correlations in the $21^{\text {st }}$ century}

\author{
Kamille Abdool,1 Karan Seegobin, ${ }^{1}$ \\ Kanterpersad Ramcharan, 1 \\ Adrian Alexander, 1 \\ Leandra Julien-Legen, 1 \\ Stanley Lawrence Giddings, 2 \\ Samuel Aboh, 2 Fidel Rampersad ${ }^{3}$ \\ 1Neurology Unit, 2Infectious Disease Unit, \\ San Fernando Teaching Hospital, \\ University of the West Indies; ${ }^{3}$ Radiology \\ Department, University of the West \\ Indies, Trinidad and Tobago
}

\begin{abstract}
We report a case of a 46-year-old man presenting with a progressive cognitive decline, ataxic gait, urinary incontinence for 4 months and neuroimaging consistent with normal pressure hydrocephalus. The atypical presentation of a progressively worsening dysphasia and a right hemiparesis dismissed as a vascular event 1 month earlier associated with normal pressure hydrocephalus prompted further investigations confirming neurosyphilis also manifesting as dementia paralytica. Treatment using consensus guidelines led to resumption of activities of daily living. Neurosyphilis, considered rare in the neuroimaging era, must still be considered a reversible cause of dementia and other neurological manifestations in contemporary neurological practice.
\end{abstract}

\section{Introduction}

Neurosyphilis has a special place in the history of Neuropsychiatric Diseases and the unraveling of the etiology, pathogenesis and control remains one of the instructive lessons in the principles and practice of medicine. Its propensity to affect all social classes is intricately linked to it being a sexually transmitted disease. The ravages left in its wake as it spread across Europe and the subsequent fascinating story of the discovery of penicillin leading to a treatment for syphilis gave impetus for its eradication in the second half of the nineteenth century.1,2 Despite some measure of success in the control and eradication, neurological presentations still occur and can pose a diagnostic dilemma to physicians. ${ }^{3-5}$ As this illness has become rare, opportunities for modern day neuroimaging are few. We report a case presenting as right sided limb weakness, facial numbness and slurred speech five months prior, diagnosed as a computed tomography (CT) scan negative vascular event in which there was subsequent deterioration leading to admission to hospital with features diagnostic of normal pressure hydrocephalus. Additionally, clinical findings consistent with dementia paralytica were present and this combination together with CT and magnetic resonance imaging (MRI) of the modern era are uncommon in the medical literature. Furthermore, to the best of our knowledge, there have been only seven reported cases of neurosyphilis presenting as normal pressure hydrocephalus..$^{3-8}$ We report the $8^{\text {th }}$ case.

\section{Case Report}

A 46-year-old male presented the emergency department with a history of a decline in his mental status and reduced level of cognition for four months. The history had to be elicited from a relative. The symptoms were associated with an unsteady gait of and urinary incontinence. He sought medical care five months prior to his presentation for weakness of the right side involving right upper and lower limbs associated with facial numbness and slurred speech. The CT brain at that time showed diffuse atrophic and small vessel changes with mild hydrocephalus (Figure 1A). He was suspected to have an atherosclerotic cerebrovascular accident and was treated empirically with aspirin, simvastatin and atenolol. As his condition was worsening he was brought to the Emergency Room, 5 months later. He had a progressive decline in his level of cognition, speech and gait was unable to express himself to the extent where his answers became yes or no responses. He became unable to care for himself independently. There was also significant weight loss of 20 pounds over the preceding three months without any change in appetite. He had no significant medical history. His prior medication included aspirin, atenolol, simvastatin, which was started since the presumed cerebrovascular event, associated with mild dyslipidemia and hypertension. The family history was noncontributory. However, he had a 20-pack year history of smoking but stopped one year prior, and a history of alcohol use for 25 years. There was no history of herbal, marijuana, cocaine or other illicit drug use. There were no recent ill contacts or travel, and he never received blood transfusions or tattoos. His relatives reported that he only had one sexual partner with whom he had three children and no history of prior sexual transmitted conditions.
Correspondence: Kanterpersad Ramcharan, Neurology Unit, San Fernando Teaching Hospital, San Fernando, Trinidad and Tobago.

Tel: +1.868 .342 .6833 . Fax: +1.868 .657 .5951 .

E-mail: kramcharan79@yahoo.com

Key word: Neurosyphilis; Neuroradiology; Normal Pressure Hydrocephalus; Syphilis.

Acknowledgements: We thank the Radiology and Photography Departments, San Fernando Teaching Hospital for preparation of the images. We thank the patient for allowing us to report his clinical data.

Contributions: the authors contributed equally.

Conflict of interest: the authors declare no potential conflict of interest.

Received for publication: 31 July 2016

Revision received: 4 August 2016.

Accepted for publication: 8 August 2016.

This work is licensed under a Creative Commons Attribution NonCommercial 4.0 License (CC BYNC 4.0).

(C) Copyright K. Abdool et al., 2016

Licensee PAGEPress, Italy

Neurology International 2016; 8:6812

doi:10.4081/ni.2016.6812

On examination he was not in respiratory distress. He was anicteric, acyanotic, afebrile and his mucus membranes were pink and moist. The pupils were equal round and reactive to light. He had a Glasgow Coma Scale of $12 / 15$ accounted by the following responses: eye $=4$, motor $=6$, verbal $=2$. His speech was limited to yes or no answers and was dysarthric. The blood pressure was 120/77, pulse 65 , respiratory rate 20 , percutaneous oxygen saturation $99 \%$, random blood glucose 147. The mini mental state examination could not be completed. He had a reduced power of $4 / 5$ on the right upper and lower limb, with associated past pointing and intention tremor. The patient was able to understand instructions but had severe difficulty executing them as well as expressing himself. His reflexes were +++ in S1S2, L3L4, C5C6 and T1T2 on both right and left sides. Plantars were upgoing on the right and equivocal on the left. There were involuntary jerky myoclonic movements of both upper and lower limbs, which were worse with movement. Sensory functions were intact. He had an ataxic broad based gait, and needed assistance while walking. Fundoscopy examination was normal. Other aspects of the physical examination were noncontributory.

Initial laboratory investigations showed normal renal function, liver function, thyroid function, C-reactive protein test, coagulation pro- 
file, electrolytes, serum B12 and red cell folate, HIV, hemoglobin electrophoresis, as well as a normal connective tissue screen. Prothrombotic screening was considered unnecessary. The electrocardiogram was in normal sinus rhythm at a rate of $50 \mathrm{bpm}$. The echocardiogram was normal with an EF 70\% with no aortic regurgitation or aortitis/ aneurysm. Electroencephalography showed subcortical dysfunction in left frontal lobe, accompanied by cortical irritability in the same area spreading posteriorly to central and temporal regions. He had mild elevation of lowdensity lipoprotein and was normotensive during his hospital stay.

CT brain on admission showed dilated ventricles with an Evans index of 0.523 out of proportion to the degree of cerebral atrophy and hypodensities in bifrontal lobes, which appeared to be consistent with edema and microangipathic small vessel disease (Figure 1B).

MRI imaging on T1,T2,T2 FLAIR, apparent diffusion coefficient (ADC) and diffusionweighted imaging (DWI) on admission confirmed hydrocephalus, bifrontal white matter hyperintensities, mesotempoaral atrophy and absence of infarctions or gumma (Figure 2A-E).

Lumbar puncture on presentation and 3 months later showed elevated protein and serological evidence confirming neurosyphilis, with improvement at 3 months following treatment (Table 1).

He was treated with aqueous crystalline penicillin G 4 million units intravenously every four hours, for 14 days. Prior to treatment he had a Barthel scale 20 and modified Rankin scale of 4 . At the end of 2 weeks of treatment his Barthel scale and Rankin scale were 90 and 3 respectively. With improved verbal communication from the patient with treatment, he reported having multiple prior sexual partners, and did not use barrier contraception on all occasions. Furthermore, he recalled having an ulcer on his penis several years prior however did not seek medical care. He had no history of secondary syphilis.

Repeat MRI at 6 months post treatment showed significant reversal of MRI findings. (Figure 3A and B)

\section{Discussion}

The clinical manifestations of neurosyphilis can occur at any stage of the infection and include asymptomatic neurosyphilis, acute meningeal syphilis, meningovascular syphilis, paretic neurosyphilis, and tabetic neurosyphilis. 9,10
Clinical features of the general paresis type are divided into early and late onset features, some of which can be seen in normal pressure hydrocephalus (NPH) which presents with ataxia, urinary incontinence and dementia. ${ }^{6,10}$ Early symptoms of the paretic type syphilis include irritability, forgetfulness, personality changes, headaches, and changes in sleep habits. Late symptoms include emotional lability, impaired memory and judgment, disorientation, confusion, delusions, and occasionally seizures. Psychiatric manifestations range from depression, to delirium (hallucinations), mania, and psychosis. Common neurological signs include pupillary abnormalities (ArgyllRobertson pupils occur in late paresis but are more often observed in tabes dorsalis,

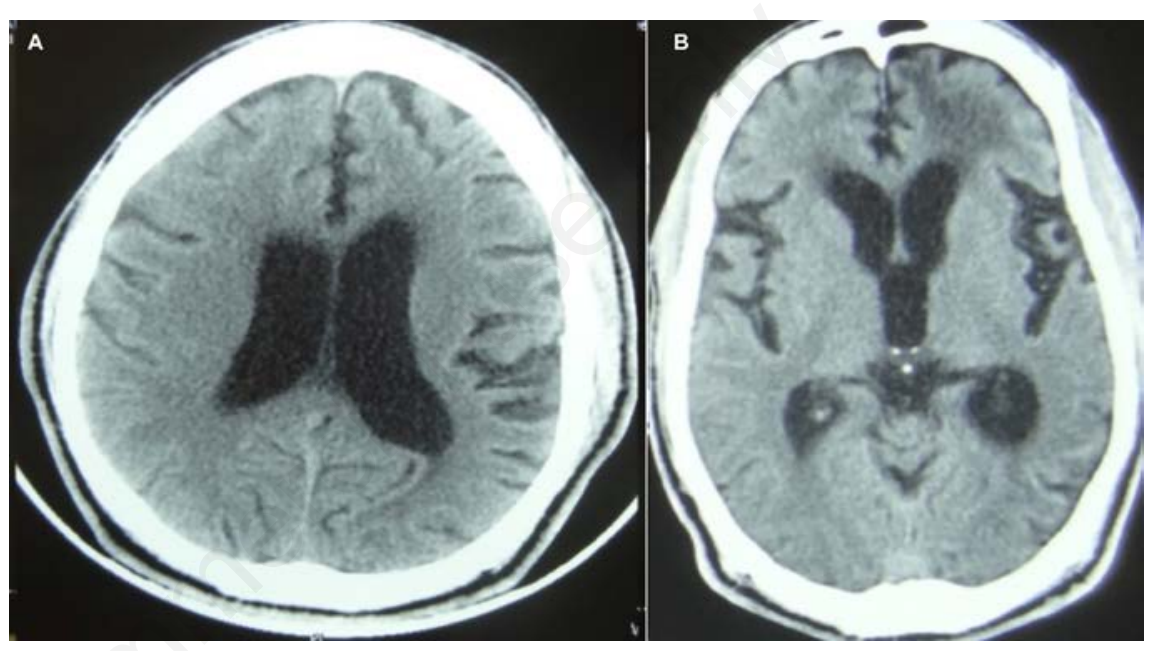

Figure 1. A) Axial non contrast computed tomography (CT) brain done five months prior to presentation showing cortical atrophy and hydrocephalus; B) axial non contrast CT brain on admission again showing hydrocephalus, with bilateral temporal atrophy, with hypodensities in the subcortical frontal region of both hemispheres, but more prominent on the left than right.

Table 1. Lumbar puncture on presentation and 3 months after treatment.

\begin{tabular}{lcc} 
& Findings on admission & Findings at 3 months after treatment \\
CSF pressure & $13.5 \mathrm{mmHg}(8-15)$ & $17.0 \mathrm{mmHg}(8-15)$ \\
CSF protein & $68.5 \mathrm{mg} / \mathrm{dL}(5-40)$ & $46.8 \mathrm{mg} / \mathrm{dL}(5-40)$ \\
\hline CSF glucose & $73 \mathrm{mg} / \mathrm{dL}(50-80)$ & $63 \mathrm{mg} / \mathrm{dL}(50-80)$ \\
CSF VDRL & Positive $1: 256$ & Positive $1: 16$ \\
\hline CSF treponema pallidum levels & $3.87(0-1.0)$ & $\mathrm{ND}$ \\
CSF FTA-ABS & Reactive & ND \\
CSF cell count & Nil & Nil \\
CSF gram stain, india ink acid fast, culture & Negative & ND \\
\hline CSF HTLV 1 and 2 & Negative & ND \\
Serum VDRL & Positive 1:1024 & ND \\
\hline Oligoclonal bands & 8 oligoclonal bands seen in CSF were & ND \\
\end{tabular}

CSF, cerebrospinal fluid; VDRL, Venereal Disease Research Laboratory; ND, not done; FTA-ABS, fluorescent treponemal antibody absorption; HTLV, human T-lymphotropic virus. 
dysarthria, and tremors of facial, lingual, and hand muscles. Rarely, optic atrophy and ocular muscle palsies were also described. Pathologically, there is atrophy of the frontal and temporal lobe with sparing of the motor, sensory, and occipital cortex. This leads to dilatation of the lateral ventricles. The Lissauer form of the disease, which occurs much less frequently, may also affect the cerebellum and basal ganglia. The average time from infection to onset of paretic neurosyphilis is between 15 and 20 years. Most untreated patients die with- in 5 years after the onset of symptoms. 10

To the best of our knowledge there have been only seven cases of neurosyphilis presenting as NPH in the literature. ${ }^{6-8}$ Since 1940 , limited case reports have encompassed the study of neurosyphilis in the post-penicillin era. Diagnosis requires clinical documentation of one or more of the characteristic symptoms of idiopathic NPH in combination with a brain imaging study demonstrating non-obstructive ventricular enlargement disproportionate to cerebral atrophy. ${ }^{9}$ Our patient satisfied the clinical and radiological criteria for $\mathrm{NPH}$, which was further confirmed with normal cerebrospinal fluid (CSF) opening pressures and positive serum and CSF serology. The possible vascular event 5 months before may have been due to vasculitis. Unfortunately, syphilis was not considered then.

CT findings of patients with neurosyphilis are not well documented and a there are few reports on the MRI findings of this disease. 5 In one report meningovascular syphilitic lesions appeared as hyperintense on MRI not seen on
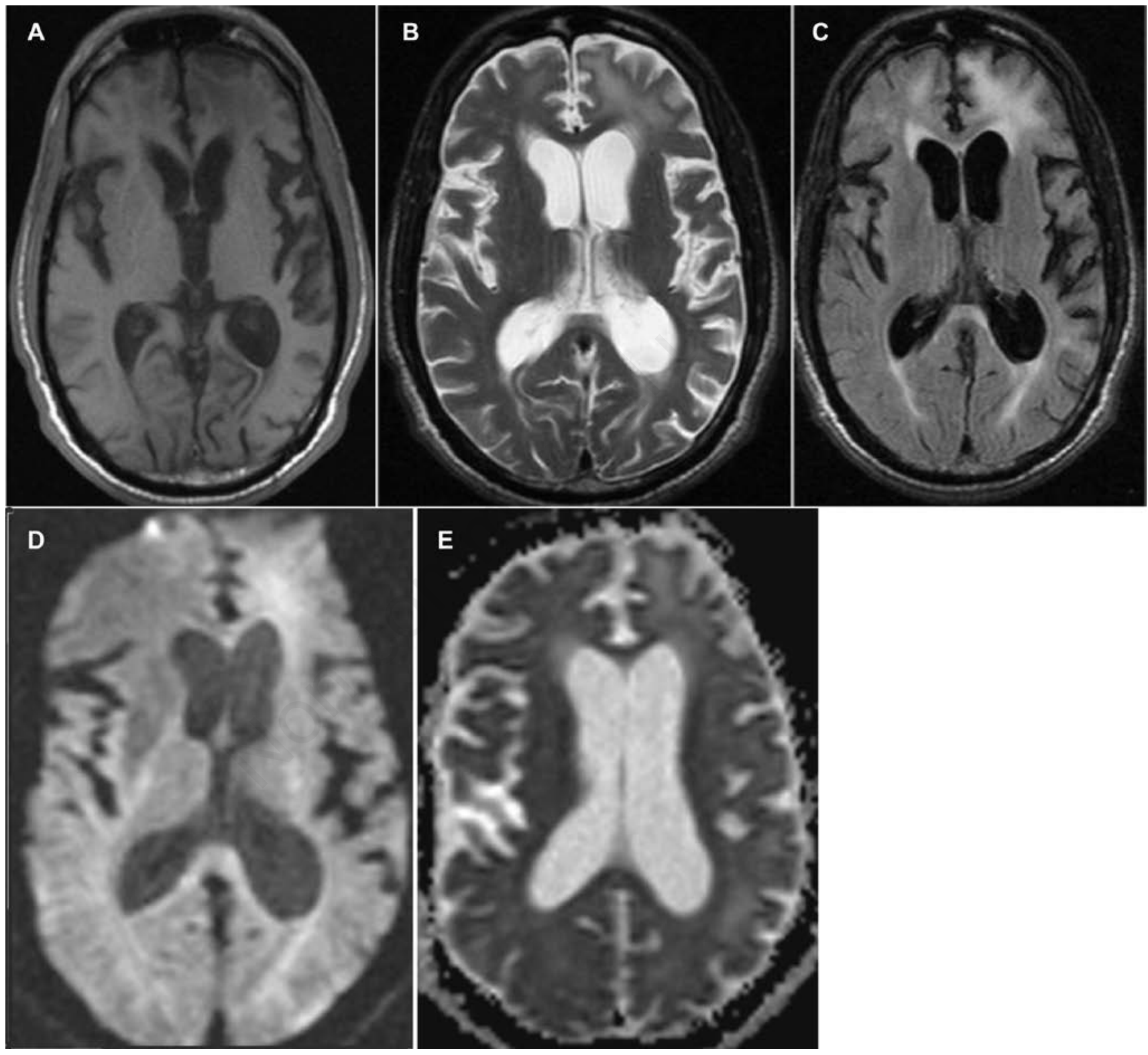

Figure 2. A) Axial T1 weighted magnetic resonance imaging (MRI) brain with temporal lobe atrophy and subcortical hypointensities in the right and left frontal regions; B) axial T2 weighted MRI brain showing dilated lateral ventricles out of proportion to the degree of cerebral atrophy, associated with hyperintensities in the left frontal subcortical region, the left parieto-occipital subcortical region, and to a lesser extent the periventricular region of the anterior horn of the right lateral ventricle; C) axial T2 FLAIR MRI brain showing bifrontal and periventricular hyperintensities; D) axial diffusion-weighted imaging MRI brain showing restricted diffusion in the left subcortical frontal lobe; E) axial apparent diffusion coefficient MRI brain showing diffuse hyperintensities in bifrontal region (left greater than right). 
CT. 5 Our microbiological studies illustrated clear confirmation.

MRI is the most sensitive technique in detecting changes in cerebral vasculitis involving large, medium, and small vessels. Its sensitivity approaches $100 \%$. Most common MRI diagnostic features in neurosyphilis are medium contrast enhancement, atrophy, white matter lesions, cerebral infarction, edema. ${ }^{11}$ In one study of patients with paretic neurosyphilis, the most common findings were generalized cerebral atrophy and foci of increased signal intensity on T2 weighted images. There was a significant association between the presence of frontal lesions and overall degree of psychiatric morbidity. 10 Neurovasculitis in MRI usually presents with focal areas of high signal intensity. Those may be observed at any distribution, including subcortical white matter, deep white matter, or gray matter with areas of infarction and hemorrhage. Parenchymal and meningeal contrast minimum enhancement can also be demonstrated. Also, multiple areas of infarction in different vascular regions and of different ages are suggestive of cerebral vasculitis. ${ }^{11}$ Common findings include hypointensity on T1, hyperintensity on $\mathrm{T} 2$, medium contrast enhancement, perilesional edema, meningeal enhancement/dural tail, T1 isointense lesion, T2 isointense lesion, T2 hypointense lesion, cerebral infarction, arteritis, nonspecific white matter lesions involving the frontotemporal lobes, hippocampus and periventricular area, atrophy, gummas, extraaxial enhancement, high signal changes in the bilateral mesial temporal lobes including both hippocampus and amygdala in T2-images and FLAIR and DWI sequence, ventricular dilatation. ${ }^{11}$ In neu- rosyphilis medium and small vessels are usually involved. The most frequently involved arteries are middle cerebral artery and branches of the basilar artery.11 Our patient had hypointensity on T1 in the left frontal region with hyperinternsity on the left frontal T2 MRI with restricted diffusion on DWI image, which support a vasculitic expression of the disease.

The goal of therapy is to inhibit the progression of the infection and reverse the symptoms. Furthermore response to therapy depends on the stage of infection. ${ }^{10}$ Antibodies produced in response to the syphilis antigen results in neuroinflammation, which can contribute to initiation and maintenance of chronic inflammation and tissue damage in neurosyphilis independent of active infection. ${ }^{4}$ Data on the treatment of psychiatric symptoms associated with paretic neurosyphilis are very limited. For late disease, especially the parenchymatous forms, resolution of symptoms may not occur. However with treatment there can be considerable regression of the neurological and psychological signs as in our patient.10-13 Repeat lumbar puncture at 3 months showed a fall in protein and VDRL titre correlating with clinical and radiological improvement.

\section{Conclusions}

A high index of clinical suspicion is required to diagnose neurosyphilis and commence treatment early. ${ }^{14}$ In a illustrative study of 116 patients with general paresis in China, after the initial evaluation, neurosyphilis was not suspected in 36 percent of patients, which delayed the diagnosis for 1 to

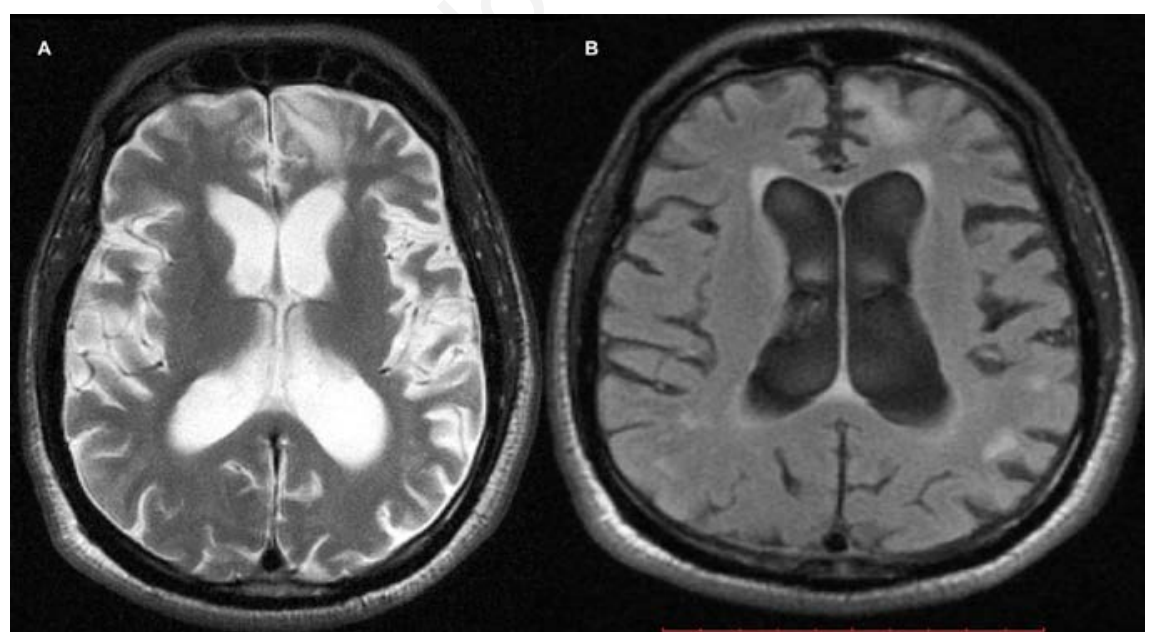

Figure 3. A) Axial T2 weighted magnetic resonance imaging (MRI) brain showing less lateral ventricualr dilation, compared to previous MRI; B) axial FLAIR MRI brain showing partial resolution of the left frontal subcortical hyperintensity, and much less periventricular hyperintensities.
24 months. ${ }^{15}$ Despite the drastic reduction in the incidence of neurosyphilis with the advent of effective diagnosis and treatment, there exists a rise in the number of cases of primary and neurosyphilis. $3,5,16,17$ These trends suggest a failure of prevention. However, the primary and secondary (P\&S) syphilis rates have increased almost every year since 2000-2001. In 2014, a total of 19,999 P\&S syphilis cases were reported, and the national P\&S syphilis rate increased to 6.3 cases per 100,000 population, the highest rate since 1994. During 2013-2014, according to the Centers for Disease Control and Prevention, the number of cases of late and late latent syphilis increased 7.9\% (from 21,819 to 23,541 cases). 16

Therefore, syphilis remains a global health issue and clinicians are reminded to be vigilant since treatment of this reversible cause of dementia can be most rewarding.

\section{References}

1. Pearce JMS. Brain disease leading to mental illness: a concept initiated by the discovery of general paralysis of the insane. Eur Neurol 2012;67:272-8.

2. Mehrabian S, Raycheva M, Traykova M, et al. Neurosyphilis with dementia and bilateral hippocampal atrophy on brain magnetic resonance imaging. BMC Neurology 2012; $12: 96$.

3. Lowenstein DH, Mills C, Simon RP. Acute syphilitic transverse myelitis: unusual presentation of meningovascular syphilis. Genitourin Med 1987;63:333-8.

4. Drago F, Javor S, Parodi A. Neurosyphilis: from infection to autoinflammation? Int $\mathrm{J}$ STD AIDS 2016;27:327-8.

5. Ito M, Sato K, Tada H, Kuru Y. Neurosyphilis manifesting as a focal mass lesion: computed tomographic and magnetic resonance imaging features-case report. Neurol Med Chir (Tokyo) 1990;30: 194-7.

6. Mok SR, Punjabi V, Shklar DL. Neurosyphillis presenting with normal pressure hydrocephalus in a 76 year old man. Am J Case Rep 2012;13:173-6.

7. De Souza RKM, Zatera A, Suzano FL, et al. Hakim-Adams syndrome secondary to syphilis. Case Rep Clin Med 2013;2:377-9.

8. Filip CR, Ungureanu A, Prodan L, et al. Neurosyphilis in a young patient presented as rapid cognitive decline. Romanian J Neurol 2014;13:216-9.

9. Tsakanikas D, Relkin N. Normal pressure hydrocephalus. Semin Neurol 2007;27:5865.

10. Ghanem KG. Review: neurosyphilis: a historical perspective and review. 
CNS Neurosci Ther. 2010;16:157-68. 11. Czarnowska-Cubałal M, Wiglusz MS, Cubała WJ, et al. MR findings in neurosyphilis-a literature review with a focus on a practical approach to neuroimaging. Psychiatr Danub 2013;25:S153-7.

12. Pedersen KK, Sorensen PS, Ankerhus J, et al. Syphilitic normal pressure hydrocephalus. Acta Neurochir 1979;48:35-9.

13. Lamy C, Eymard B, Léger JM, et al.
Communicating hydrocephalus disclosing neurosyphilis. Rev Neurol 1990; 146:54-6.

14. Kayal AK, Goswami M, Das M, et al. Clinical spectrum of neurosyphilis in North East India. Neurol India 2011;59:344-50.

15. Zheng D, Zhou D, Zhao Z, et al. The clinical presentation and imaging manifestation of psychosis and dementia in general paresis: a retrospective study of 116 cases.
J Neuropsychiatry Clin Neurosci 2011; 23:300-7.

16. Centers for Disease Control and Prevention. 2014 Sexually transmitted disease surveillance. Available from: http://www.cdc.gov/std/stats14/default.htm.

17. Drago F, Merlo G, Ciccarese G, et al. Changes in neurosyphilis presentation: a survey on 286 patients. Eur Acad Dermatol Venereol 2016. [Epub ahead of print] 